\title{
The IGA IIPG on the Union of Overlapped Patches
}

\author{
Honghai Zhang, Rong Mo and Neng Wan \\ Key Laboratory of Contemporary Design and Integrated Manufacturing Technology, Ministry of Education, Northwestern \\ Polytechnical University, Xi’an, China, 710072
}

\begin{abstract}
Recently, an IGA DG method (IGAODG) is developed to solve PDEs defined on the overlapped patches. With IGAODG, the time-consuming treatments for keeping the integration precision on the trimmed patches are avoided. In this paper, the IGAODG is extended with the incomplete interior penalty method (IIPG), and the discretization scheme for the Poisson's equation is created and tested. Numerical results show that the presented method will give the optimal convergence orders.
\end{abstract}

Keywords-isogeometric analysis; discontinuous galerkin method; overlapped domain; IGAODG

\section{INTRODUCTION}

The Isogeometric Analysis (IGA ,Hughes et al.[1]-[4]) applies the NURBS basis to describe both the geometries and the unknown fields, and it is efficient to realize h-, p-, krefinements[4], [5] with which the refined geometries are kept unchanged exactly. Now, IGA has been successfully applied to solve many physical problems, such as the elasticity, the electromagnetism, the fluid-structure interaction, and the shape optimization problems. When the domain is the union of multiple patches, three kinds of IGA methods can be applied:

- The first kind of IGA methods requires the matched or mismatched patches to be $\mathrm{C}^{0}$-continuous[6], [7]. That is, the patches should be carefully designed to contact with each other, otherwise the non-watertight geometries will be generated.

- The second kind[8]-[10] usually supports the union, subtraction and intersection operations. Because the three operations are based on the trimming algorithms, suitable integration schemes should be applied to maintain the integration precision on the trimmed patches. These integration schemes, for example the adaptive octree decomposition method, are often time consuming.

- The third kind allows the overlapped and non-trimmed patches. In reference[11] , the Additive Schwarz Domain Decomposition Method (ASDDM) is applied to solve PDEs on overlapped domains. With this method, the united patches needs not to be trimmed, so that it does not require additional treatments in the integration process. However, with this DD method, the overlapped boundaries must be assigned as the external boundary of a sub-domain. This may affect the efficiency of the DD method. In reference[12], an IGA DG method (IGAODG) is developed to solve problems defined on the united patches also.
The DG methods are widely applied and deeply researched. Simply speaking, for the boundary value problem

$$
\begin{aligned}
& -\Delta u=f \quad \text { in } \Omega \\
& \left.u\right|_{\partial \Omega}=0
\end{aligned}
$$

where the domain is meshed into the non-overlapped quadrilateral elements ( $T_{h}$ in Figure I), the symmetric interior penalty method (SIPG) of problem(1) reads as:

Find $u_{h} \in V_{h}$ such that

$$
\begin{aligned}
\int_{T_{h}} f v d x & =\int_{T_{h}} \nabla u_{h} \cdot \nabla v d x-\int_{\Gamma}[v]\left\{\nabla u_{h}\right\}+\left[u_{h}\right]\{\nabla v\} d s \\
& +\int_{\Gamma} \alpha_{e}\left[u_{h}\right][v] d s, \quad \forall v \in V_{h}
\end{aligned}
$$

While with the IIPG, (2) should be replaced by

$$
\begin{aligned}
\int_{T_{h}} f v d x & =\int_{T_{h}} \nabla u_{h} \cdot \nabla v d x-\int_{\Gamma}[v]\left\{\nabla u_{h}\right\} d s \\
& +\int_{\Gamma} \alpha_{e}\left[u_{h}\right][v] d s, \quad \forall v \in V_{h}
\end{aligned}
$$

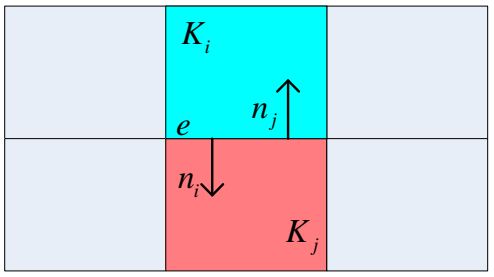

FIGURE I.

A DOMAIN IS MESHED INTO QUADRILATERAL ELEMENTS.

$\operatorname{In}(2)$ and(3), for a scalar function $v$ or a vector function $\tau$, the average operator $\{$.$\} and jump operator [.] on the element$ boundaries are defined by

$$
\begin{aligned}
& \text { on } e \not \subset \partial \Omega:\left\{\begin{array}{l}
\{v\}=0.5\left(v_{i}+v_{j}\right),[v]=v_{i} n_{i}+v_{j} n_{j} \\
\{\tau\}=0.5\left(\tau_{i}+\tau_{j}\right),[\tau]=\tau_{i} \cdot n_{i}+\tau_{j} \cdot n_{j}
\end{array},\right. \\
& \text { on } e \subset \partial \Omega:\left\{\begin{array}{l}
{[v]=v n} \\
\{\tau\}=\tau
\end{array}\right.
\end{aligned}
$$


and $\alpha_{e}:=\eta_{e} / h_{e}$ is the penalty coefficient where $\eta_{e}$ is a positive number large enough and $h_{e}$ is the size of the concerned boundary.

IGAODG is developed under the SIPG concept. However, the stiffness matrix is not symmetric for the reason to avoid integrations on the trimmed boundaries. Therefore, the IGAODG is suitable for the relatively small problems or to be used as a subroutine of a DD method. In this paper, the original IGAODG is extended with IIPG to reduce some of the computations costs for assembling the matrix equations, and the discretization schemes of this IIPG extension are created for the Poisson's equations. As a part of the IGAODG research, results show that this extension also gives the optimal convergence speed.

This paper is arranged as follows. After the introductions in section I, we briefly introduce the concept of IGA and give some requisite notations for overlapped patches in section II. In section III, we create the discretization scheme for the Poisson's equation. In section IV, the convergence property is tested numerically. Finally, the paper is summarized in section V.

\section{IGA AND SOME NOTATIONS}

In the following discussions, the hat ' $\wedge$ ' is used to indicate that a function or a space is defined on the parametric domain. Between the parametric patch $\hat{P}$ and the physical patch $P$, a NURBS-based geometry can be seen as a map $F: \hat{P} \rightarrow P$. Therefore, a discrete function space on the physical patch can be defined with

$$
S^{h}(P):=\operatorname{span}\left\{\hat{B}_{i} \circ F^{-1}\right\}
$$

where $\hat{B}_{i}$ is the $i$-th NURBS basis. On a parametric patch, the open knot vectors indicate a mesh, marked as $M_{p r}(\hat{P})$, so that on the physical patch, a physical mesh $M_{p r}(P)$ can be defined by

$$
M_{p r}(P)=\left\{K \mid K=F(\hat{K}), \hat{K} \in M_{p r}(\hat{P})\right\}
$$

Defining a function $\delta_{K}$ by

$$
\delta_{K}=\left\{\begin{array}{lc}
0, & x \notin K \\
1, & \text { else }
\end{array},\right.
$$

the space $S^{h}(P)$ can be further broken into a space $S_{K}^{h}(P)$ where

$$
S_{K}^{h}(P):=\operatorname{span}\left\{v_{K}^{h} \mid v_{K}^{h}=\delta_{K} v^{h}, \forall v^{h} \in S^{h}(P)\right\}
$$

Functions in $S_{K}^{h}(P)$ may be non-zero out of an element $K$. We use the definition (6) and the following definition (8) to avoid discussing different continuities of basis between elements. For example, some B-spline basis may be continuous between elements, while the Bezier functions are discontinuous between elements.

With these definitions, a physical mesh and a discrete physical function space are created on a patch, so that an IGA method can be developed..

We suppose that a parametric patch $\hat{P}$ is precisely meshed into a collection of elements $M_{p r}(\hat{P})=\{\hat{K}\}$. If the CAD model contains multiple patches, we denote by $\hat{T}_{h}=\bigcup_{i} M_{p r}\left(\hat{P}_{i}\right)$ the parametric mesh. Similarly, the physical mesh is defined by $T_{h}=\bigcup_{i} M_{p r}\left(P_{i}\right)$ where

$$
M_{p r}(P)=\left\{K \mid K=F(\hat{K}), \quad \hat{K} \in M_{p r}(\hat{P})\right\} .
$$

We also suppose that the meshes $T_{h}$ and $\hat{T}_{h}$ are quasiuniform, so that we can use a single symbol $h$ to represents the sizes of both meshes. For an overlapped domain shown in Figure II, the mesh refinement process is given in Figure III.

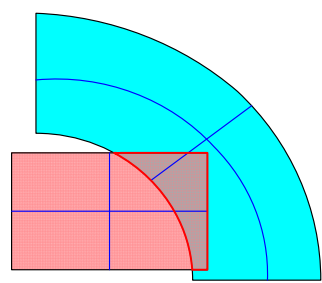

FIGURE II.

$$
\text { TWO UNITED PATCHES. }
$$

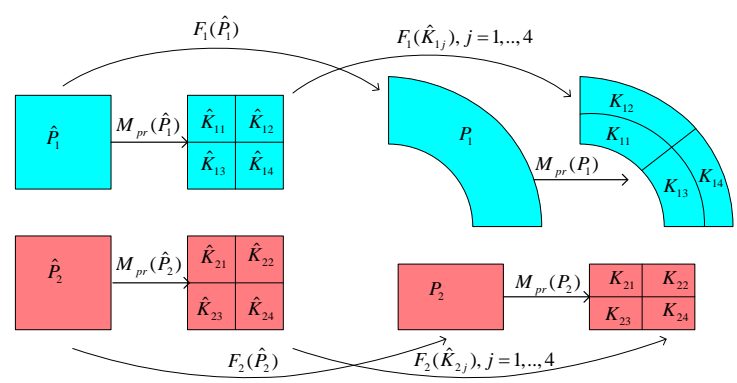

FIGURE III.

$$
\text { REFINEMENTS OF THE TWO MESHES } T_{h} \text { AND } \hat{T}_{h} \text {. }
$$

If the patches are overlapped, we need three kinds of boundary collections as defined in(7). The first collection $\Gamma_{d}$ contains the external boundaries of the whole domain $\Omega$. The second collection $\Gamma_{e}$ contains all the adjacent boundaries of brother elements in their parent patches. The third collection $\Gamma_{p}$ contains all the external boundaries of patches, which do not belong to $\Gamma_{e} \cup \Gamma_{d}$. In Figure II, boundaries colored red $\subset \Gamma_{p}$, boundaries colored blue $\subset \Gamma_{e}$, and boundaries 
colored black $\subset \Gamma_{d}$. Finally, we denote by $\Gamma:=\Gamma_{d} \cup \Gamma_{e} \cup \Gamma_{p}=\bigcup_{K}(\partial K)$ the collection of all boundaries of all elements.

$$
\begin{aligned}
& \Gamma_{d}:=\bigcup_{K \in T_{h}}(\partial \Omega \cap \partial K) \\
& \Gamma_{e}:=\bigcup_{P}\left\{e \mid e=\partial K_{i} \cap \partial K_{j}, \forall K_{i}, K_{j} \in M_{p r}(\mathrm{P}), i \neq j\right\} \\
& \Gamma_{p}:=\bigcup_{K}(\partial K) \backslash\left(\Gamma_{e} \cup \Gamma_{d}\right)
\end{aligned}
$$

On the domain $\Omega$, seen as the non-overlapped result of the union operation, the Sobolev function space $H^{k}(\Omega)$ and its norm are defined as usual. In the space $H^{k}(\Omega)$, the functions are single-valued.

On the overlapped domain, we define space $H^{k}\left(T_{h}\right)$ as follows: 1) a function $u$ on each element $K \in T_{h}$ belongs to $H^{k}(K)$, 2) the function $u_{K}$ needs not to be continuous with its brother elements, and 3 ) the function $u_{K}$ needs not to be as same as the fields on the other overlapped elements of other parent patches. In other words, a function $u \in H^{k}\left(T_{h}\right)$ may be double-valued on the overlapped parts (or the intersections). On $H^{k}\left(T_{h}\right)$, the Sobolev norm is defined by the $L^{2}$ inner product as

$$
\|u\|_{k, T_{h}}^{2}=\sum_{K \in T_{h}}\left\|u_{K}\right\|_{k, K}^{2}, \quad\left\|u_{K}\right\|_{k, K}^{2}:=\sum_{i=0}^{k}<D^{i} u, D^{i} u>_{L^{2}(K)} .
$$

Similarly, we define a discrete space $S_{K}^{h}\left(T_{h}\right) \subset H^{k}\left(T_{h}\right)$ by

$$
S_{K}^{h}\left(T_{h}\right)=\operatorname{span}\left\{v_{K}^{h} \mid v_{K}^{h}=\delta_{K} v^{h}, \forall v^{h} \in \forall S^{h}\left(P_{i}\right), \bigcup P_{i}=\Omega\right\}
$$

On the boundary $\partial K_{i}$, for a scalar function $v_{K_{i}} \in S_{K}^{h}\left(T_{h}\right)$, we define its adjacent value $v_{K_{i}, e}$ by

$$
v_{K_{i}, e}(x)=\left\{\begin{array}{l}
v_{K_{j}}(x), \quad \text { on } e \subset \Gamma_{e}, \text { if } x \in \partial K_{i} \cap \partial K_{j} \\
0, \quad \text { on } e \subset \Gamma_{d} \\
v_{K_{j}}(x), \quad \text { on } e \subset \Gamma_{p}, \text { if } x \in \partial K_{i} \cap K_{j}
\end{array} .\right.
$$

Definition(8) and (9) can be extended to their vector (tensor) cases. If no ambiguity exists, we directly reuse the symbol $S_{K}^{h}\left(T_{h}\right)$ to represent the discrete space of vector (tensor) functions and reuse $v_{K_{i}, e}(x)$ to represent the adjacent value of a vector (tensor) functions.

\section{DISCRETIZATION SCHEME}

As shown in(3), in the non-overlapped case, if we select a test function $v_{K}^{h}=\delta_{K} v^{h}, v^{h} \in S_{K}^{h}\left(T_{h}\right)$, it is indicated that the $\mathrm{C}^{0}$ and $\mathrm{C}^{1}$ - continuities are penalized by $\alpha_{e} v_{K}^{h} n_{e}$ and $\frac{v_{K}^{h}}{2}$ respectively. For example, the second item $-\int_{\Gamma}[v]\left\{\nabla u_{h}\right\} d s$ indicates the punishment on the $\mathrm{C}^{1}$ - continuity, because

$$
\begin{aligned}
-\int_{e}[v]\left\{\nabla u_{h}\right\} d s & =-\int_{e} v_{i} n_{i} \nabla u_{h, i} d s-\int_{e} v_{j} n_{j} \nabla u_{h, j} d s \\
& +\int_{e} \frac{v_{i}}{2}\left[\nabla u_{h}\right] d s+\int_{e} \frac{v_{j}}{2}\left[\nabla u_{h}\right] d s
\end{aligned} .
$$

In(10), the first two items are naturally brought by the integration by parts formula. In the following of this paper, we only discuss the punishment on the $C^{1}$-continuity, because the $\mathrm{C}^{0}$-continuity is easy to be understood.

In the overlapped case, we extend the $C^{0}$ - and $C^{1}$ punishments onto the boundaries in $\Gamma_{p}$. By applying the same penalty coefficients, $\alpha_{e} v_{K}^{h} n_{e}$ and $\frac{v_{K}^{h}}{2}$, on $\Gamma_{p}$, the IGA IIPG for problem (1) defined on the overlapped domain reads as:

Find $u^{h} \in S_{K}^{h}\left(T_{h}\right)$ such that

$$
\sum_{K} \int_{K} f v_{K}^{h} d x=\sum_{K} B_{K}\left(u^{h}, v_{K}^{h}\right), \forall v_{K}^{h}=\delta_{K} v^{h}, v^{h} \in S_{K}^{h}\left(T_{h}\right),
$$

where

$$
\begin{aligned}
& B_{K}\left(u^{h}, v_{K}^{h}\right)=\int_{K} \nabla u_{K}^{h} \cdot \nabla v_{K}^{h} d x \\
& \quad-\int_{\partial K \cap \Gamma_{e}} v_{K}^{h} n_{k} \nabla u_{K}^{h} d s+\int_{\partial K \cap \Gamma_{e}} \frac{1}{2} v_{K}^{h}\left(\nabla u_{K}^{h}-\nabla u_{K, e}^{h}\right) n_{k} d s \\
& +\int_{\partial K \cap \Gamma_{e}}\left(\alpha_{e} n_{k} v_{K}^{h}\right)\left(u_{K}^{h}-u_{K, e}^{h}\right) n_{k} d s \\
& \quad-\int_{\partial K \cap \Gamma_{d}} v_{K}^{h} n_{k} \nabla u_{K}^{h} d s+\int_{\partial K \cap \Gamma_{d}}\left(\alpha_{e} n_{k} v_{K}^{h}\right)\left(u_{K}^{h}-u_{K, e}^{h}\right) n_{k} d s \\
& \quad-\int_{\partial K \cap \Gamma_{p}} v_{K}^{h} n_{k} \nabla u_{K}^{h} d s+\int_{\partial K \cap \Gamma_{p}} \frac{1}{2} v_{K}^{h}\left(\nabla u_{K}^{h}-\nabla u_{K, e}^{h}\right) n_{k} d s \\
& +\int_{\partial K \cap \Gamma_{p}}\left(\alpha_{e} n_{k} v_{K}^{h}\right)\left(u_{K}^{h}-u_{K, e}^{h}\right) n_{k} d s
\end{aligned}
$$

$\operatorname{In}(11), \delta_{K}$ is removable and it is used to emphasize that the presented method can be applied on elements of different continuities.

\section{NuMERICAL EXAMPLES}

We use (11) to solve the problem 


$$
\begin{aligned}
& \Delta u=-\sin (x)-\cos (x)-\sin (y)-\cos (y) \\
& \left.u\right|_{\partial \Omega}=\sin (x)+\cos (x)+\sin (y)+\cos (y)
\end{aligned},
$$

where the overlapped domain (the union of two squares) is given in Figure IV. We construct these two squares with identity maps.

Table I and II list the computation results with different polynomial orders and penalty coefficients. In the tables, $n$ denotes the variable numbers, $e_{i, j}$ (titled as $e_{i}$ ) denotes the error $\left\|u-u^{h}\right\|_{i, T_{h}} \quad$ at the $j^{\text {th }}$ refinement, and $R_{i}=\ln \left(\frac{e_{i, j}}{e_{i, j+1}}\right) / \ln \left(\frac{h_{j}}{h_{j+1}}\right)$ denotes the convergence order. In this paper, a centrally splitting refinement method is applied on the parametric mesh. That is, the B-spline patches are firstly refined into Bezier elements and then in further refinement, a Bezier element is centrally refined into four Bezier subelements. We also apply a single penalty coefficient $\alpha_{e}$ for each boundary of $\Gamma$. After each refinement step, $\alpha_{e}$ is doubled.

From table I and table II, it can be seen that, for most of the given penalty coefficients, the numerical solutions are convergent with the optimal orders, that is, $R_{0} \approx p+1$ and $R_{1} \approx p$. We remark here that, the method is tested on other overlapped and non-identity-mapped patches, and the same optimal convergence orders are obtained.

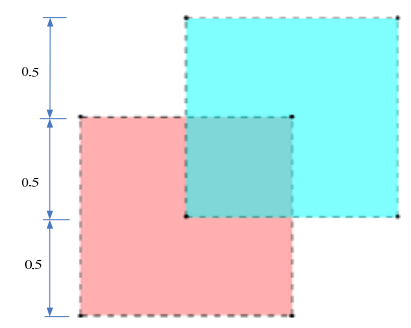

FIGURE IV.

THE UNION OF TWO OVERLAPPED SQUARES

TABLE I. RESULTS FOR POISSON EQUATION WITH P=2

\begin{tabular}{|c|c|c|c|c|c|}
\hline $\begin{array}{c}\text { Penalty } \\
\text { Ceof. }\end{array}$ & $\mathbf{n}$ & $\mathbf{R}_{\mathbf{0}}$ & $\mathbf{R}_{\mathbf{1}}$ & $\mathbf{e}_{\mathbf{0}}$ & $\mathbf{e}_{\mathbf{1}}$ \\
\hline \multirow{4}{*}{$1 \mathrm{e} 3$} & 18 & 2.880 & 1.980 & 0.00599 & 0.053295 \\
\cline { 2 - 6 } & 72 & 2.971 & 1.923 & $8.13 \mathrm{E}-04$ & 0.013509 \\
\cline { 2 - 6 } & 288 & 2.992 & 2.033 & $1.04 \mathrm{E}-04$ & 0.003563 \\
\cline { 2 - 6 } & 1152 & - & - & $1.30 \mathrm{E}-05$ & $8.71 \mathrm{E}-04$ \\
\hline \multirow{4}{*}{$1 \mathrm{e} 4$} & 18 & 2.883 & 1.980 & 0.005964 & 0.053235 \\
\cline { 2 - 6 } & 72 & 2.973 & 1.922 & $8.08 \mathrm{E}-04$ & 0.013498 \\
\cline { 2 - 6 } & 288 & 2.993 & 2.033 & $1.03 \mathrm{E}-04$ & 0.003563 \\
\cline { 2 - 6 } & 1152 & - & - & $1.29 \mathrm{E}-05$ & $8.70 \mathrm{E}-04$ \\
\hline \multirow{4}{*}{$1 \mathrm{e} 5$} & 18 & 2.884 & 1.980 & 0.005961 & 0.053229 \\
\cline { 2 - 6 } & 72 & 2.973 & 1.922 & $8.08 \mathrm{E}-04$ & 0.013497 \\
\cline { 2 - 6 } & 288 & 2.993 & 2.033 & $1.03 \mathrm{E}-04$ & 0.003563 \\
\cline { 2 - 6 } & 1152 & - & - & $1.29 \mathrm{E}-05$ & $8.70 \mathrm{E}-04$ \\
\hline \multirow{4}{*}{$1 \mathrm{e} 6$} & 18 & 2.884 & 1.980 & 0.005961 & 0.053228 \\
\cline { 2 - 6 } & 72 & 2.973 & 1.922 & $8.08 \mathrm{E}-04$ & 0.013497 \\
\cline { 2 - 6 } & 288 & 2.993 & 2.033 & $1.03 \mathrm{E}-04$ & 0.003563 \\
\cline { 2 - 6 } & 1152 & - & - & $1.29 \mathrm{E}-05$ & $8.70 \mathrm{E}-04$ \\
\hline
\end{tabular}

TABLE II. RESULTS FOR POISSON EQUATION WITH P=3

\begin{tabular}{|c|c|c|c|c|c|}
\hline $\begin{array}{c}\text { Penalty } \\
\text { Ceof. }\end{array}$ & $\mathbf{n}$ & $\mathbf{R}_{\mathbf{0}}$ & $\mathbf{R}_{\mathbf{1}}$ & $\mathbf{e}_{\mathbf{0}}$ & $\mathbf{e}_{\mathbf{1}}$ \\
\hline \multirow{4}{*}{$1 \mathrm{e} 3$} & 32 & 4.045 & 3.063 & $9.58 \mathrm{E}-04$ & 0.016537 \\
\cline { 2 - 6 } & 128 & 4.005 & 3.014 & $5.81 \mathrm{E}-05$ & 0.001979 \\
\cline { 2 - 6 } & 512 & 3.993 & 3.004 & $3.62 \mathrm{E}-06$ & $2.45 \mathrm{E}-04$ \\
\cline { 2 - 6 } & 2048 & - & - & $2.27 \mathrm{E}-07$ & $3.05 \mathrm{E}-05$ \\
\hline \multirow{4}{*}{$1 \mathrm{e} 4$} & 32 & 4.007 & 3.061 & $9.37 \mathrm{E}-04$ & 0.016533 \\
\cline { 2 - 6 } & 128 & 3.998 & 3.015 & $5.83 \mathrm{E}-05$ & 0.001981 \\
\cline { 2 - 6 } & 512 & 3.991 & 3.005 & $3.65 \mathrm{E}-06$ & $2.45 \mathrm{E}-04$ \\
\cline { 2 - 6 } & 2048 & - & - & $2.29 \mathrm{E}-07$ & $3.05 \mathrm{E}-05$ \\
\hline \multirow{4}{*}{$1 \mathrm{e} 5$} & 32 & 4.003 & 3.061 & $9.34 \mathrm{E}-04$ & 0.016533 \\
\cline { 2 - 6 } & 128 & 3.997 & 3.015 & $5.83 \mathrm{E}-05$ & 0.001981 \\
\cline { 2 - 6 } & 512 & 3.991 & 3.005 & $3.65 \mathrm{E}-06$ & $2.45 \mathrm{E}-04$ \\
\cline { 2 - 6 } 106 & 2048 & - & - & $2.30 \mathrm{E}-07$ & $3.05 \mathrm{E}-05$ \\
\hline \multirow{4}{*}{$1 \mathrm{e} 6$} & 32 & 4.002 & 3.061 & $9.34 \mathrm{E}-04$ & 0.016533 \\
\cline { 2 - 6 } & 128 & 3.997 & 3.015 & $5.83 \mathrm{E}-05$ & 0.001981 \\
\cline { 2 - 6 } & 512 & 3.988 & 3.005 & $3.65 \mathrm{E}-06$ & $2.45 \mathrm{E}-04$ \\
\cline { 2 - 6 } & 2048 & - & - & $2.30 \mathrm{E}-07$ & $3.05 \mathrm{E}-05$ \\
\hline
\end{tabular}

\section{SUMMARY}

This paper introduced an IGA IIPG method to solve Poisson's equation which are defined on the overlapped domains. With this method, the time-consuming treatments on keeping the integration precision are avoided. Comparing with IGAODG, the presented method needs less computation cost to create the matrix equations. In our numerical results, the optimal convergence orders are obtained.

\section{ACKNOWLEDGMENT}

The project is supported by the National Natural Science Fund (Program No.51775445) and the Natural Science Basic Research Plan in Shaanxi Province of China (Program No.2016JM5040).

\section{REFERENCES}

[1] Y. Bazilevs, L. Beirão Da Veiga, J. A. Cottrell, T. J. R. Hughes, And G. Sangalli, "Isogeometric Analysis: Approximation, Stability And Error Estimates For H-Refined Meshes,” Math. Model. Methods Appl. Sci., vol. 16, no. 07, pp. 1031-1090, 2006.

[2] J. A. Cottrell, T. J. R. Hughes, and Y. Bazilevs, Isogeometric Analysis Toward Intergration of CAD and FEA. A John Wiley and Sons, Ltd., 2009.

[3] T. J. R. Hughes, J. A. Cottrell, and Y. Bazilevs, "Isogeometric analysis: CAD, finite elements, NURBS, exact geometry and mesh refinement," Comput. Methods Appl. Mech. Eng., vol. 194, no. 39-41, pp. 41354195, 2005.

[4] L. Beirão da Veiga, A. Buffa, J. Rivas, and G. Sangalli, "Some estimates for h-p-k-refinement in Isogeometric Analysis,” Numer. Math., vol. 118, no. 2, pp. 271-305, Oct. 2010.

[5] J. Cottrell, T. J. R. Hughes, and A. Reali, "Studies of refinement and continuity in isogemetric analysis," Comput. Methods Appl. Mech. Eng., vol. 196, pp. 4160-4183, 2007.

[6] U. Langer, A. Mantzaflaris, and S. E. Moore, "Multipatch Discontinuous Galerkin Isogeometric Analysis,” 2014.

[7] U. Langer and I. Toulopoulos, "Analysis of Discontinuous Galerkin IGA Approximations to Elliptic Boundary Value Problems,” math.NA, pp. 132, Aug. 2014.

[8] D. Natekar, X. Zhang, and G. Subbarayan, "Constructive solid analysis: A hierarchical, geometry-based meshless analysis procedure for integrated design and analysis," CAD Comput. Aided Des., vol. 36, no. 5 , pp. 473-486, 2004. 
[9] B.-Q. Zuo, Z.-D. Huang, Y.-W. Wang, and Z.-J. Wu, "Isogeometric analysis for CSG models,” Comput. Methods Appl. Mech. Engrg., vol. 285, pp. 102-124, 2015.

[10] Y. Wang, Z. Huang, Y. Zheng, and S. Zhang, "Isogeometric analysis for compound B-spline surfaces,” Comput. Methods Appl. Mech. Eng., vol. 261-262, pp. 1-15, 2013.

[11] M. Bercovier and I. Soloveichik, "Overlapping non Matching Meshes Domain Decomposition Method in Isogeometric Analysis,” arXiv Prepr. arXiv1502.03756, 2015.

[12] H. Zhang, R. Mo, and N. Wan, "An IGA Discontinuous Galerkin Method on the union of overlapped patches," Comput. Methods Appl. Mech. Eng., vol. 326, pp. 446-480, 2017. 